\title{
Linx
}

Revue des linguistes de l'université Paris X Nanterre

$82 \mid 2021$

Entre vieillissement et innovation : le changement linguistique

\section{La vie d'un linguiste : Jean-François Sablayrolles (1951-2020)}

John Humbley

\section{(2) OpenEdition \\ Journals}

Édition électronique

URL : https://journals.openedition.org/linx/7968

DOI : $10.4000 /$ linx.7968

ISSN : 2118-9692

Éditeur

Presses universitaires de Paris Nanterre

Référence électronique

John Humbley, «La vie d'un linguiste : Jean-François Sablayrolles (1951-2020) », Linx [En ligne], 82 |

2021, mis en ligne le 30 juin 2021, consulté le 21 juillet 2021. URL : http://journals.openedition.org/ linx/7968; DOl : https://doi.org/10.4000/linx.7968

Ce document a été généré automatiquement le 21 juillet 2021.

Département de Sciences du langage, Université Paris Ouest 


\title{
La vie d'un linguiste : Jean-François Sablayrolles (1951-2020)
}

\author{
John Humbley
}

1 Jean-François Sablayrolles n'aura pas vu ce numéro de Linx où est publié son article, mais il aurait été très heureux que son travail soit reconnu par le prestigieux établissement de l'enseignement supérieur que l'on appelait autrefois tout simplement Nanterre, car cette université représente un des deux pôles de recherche à l'origine de la néologie en tant que discipline de la linguistique moderne. Le premier artisan en la matière a été sans contestation Bernard Quemada, responsable dans un premier temps de la partie «néologie » du Trésor de la Langue Française à Besançon puis de l'ensemble de ce projet lexicographique pharaonique élaboré à Nancy. Mais dès le début des années 1970 le bouillonnement de recherches en néologie et sur des sujets proches se faisait sentir à Nanterre autour de Louis Guilbert, recherches qui se caractérisaient par leur ouverture: vers la sociolinguistique (domaine de prédilection de Christiane Marcellesi et Jean-Baptiste Marcellesi), la terminologie (Louis Guespin), les discours spécialisés (Bernard Gardin, Marie-Françoise Mortureux), l'histoire de la langue (Guilbert lui-même), pour ne mentionner que les principaux courants qui irriguaient les séminaires de linguistique, sans oublier la grande question du moment: la grammaire générative ! Mais la réflexion sur l'évolution de la langue caractérisait déjà les préoccupations de ces linguistes, préoccupation que Jean-François Sablayrolles a eu à cœur de continuer, comme le montre l'article qu'il a rédigé pour le présent volume à la demande de Gaétane Dostie sur le renouvellement du lexique comme de son vieillissement, autant d'aspects qui méritent que l'on décrive, que l'on comprenne et que l'on rende compte.

2 La vie d'un linguiste, comme celle de la langue, n'est pas nécessairement un long fleuve tranquille. Rien, a priori, rien ne destinait Jean-François Sablayrolles à se spécialiser dans la néologie ni à se donner la tâche de la réhabiliter en tant que branche légitime de la linguistique. Agrégé de grammaire, il a commencé sa carrière comme professeur dans le secondaire, mais bientôt il a éprouvé le besoin de faire des recherches qui l'ont amené, vers la fin des années 1980, à pousser la porte du Centre de terminologie et de 
néologie (unité de l'Institut national de la langue française), dirigé à l'époque par le sémanticien Pierre Lerat. La problématique de l'innovation lexicale le taraudait déjà.

3 Cette fascination s'est concrétisée grâce aux conseils de Blanche-Noëlle Grunig et Roland Grunig et c'est sous la direction de la première que sa thèse a été soutenue en 1996 à l'Université Paris 8. Thèse qui a fait date. Outre une synthèse des approches antérieures sur la néologie - dont celles des collègues nanterriens - le candidat proposait un cadre d'analyse inspiré des matrices lexicogénétiques de Jean Tournier. Le livre tiré de sa thèse a été publié dans la collection dirigée par Bernard Quemada (LEXICA : Mots et dictionnaires) aux éditions Honoré Champion ${ }^{1}$, collection bientôt reprise et animée depuis par Jean Pruvost. La rencontre avec Jean Pruvost a été suivie d'une longue et fructueuse collaboration, qui a duré toute la vie de Jean-François et qui a été inaugurée par la publication en 2003 d'un « Que sais-je ? » à succès, Les néologismes : la vie d'une langue, qui a connu trois rééditions $(2012,2016,2019)$ et qui annonçait la thématique du présent numéro.

4 Jean-François Sablayrolles a obtenu son premier poste dans le supérieur à l'Université de Limoges, où il a organisé un premier colloque international sur la néologie, rencontre mémorable qui a réuni des spécialistes de nombreux pays, surtout de langue latine. La publication des actes verra le jour en 2003 toujours chez Honoré Champion, sous le titre L'innovation lexicale.

5 Nommé à l'Université Paris-Diderot (actuellement l'Université de Paris), il y passe en 2004 son Habilitation à diriger des recherches, présentée sous le titre programmatique : La néologie, point privilégié d'observation et d'étude de la langue dans son fonctionnement, ses évolutions et ses descriptions. Il obtiendra un poste de professeur de linguistique à l'Université Paris XIII (actuellement Université Sorbonne Paris-Nord), où il développera un vaste programme de recherche et de formation doctorale au sein du Laboratoire de Linguistique Informatique (plus tard Lexiques, Dictionnaires, Informatique), en particulier en collaboration avec Emmanuel Cartier mais aussi avec une équipe de chercheurs et de doctorants auxquels il avait communiqué sa passion. Le premier étage de cette fusée néologique a été une base de données néologiques, Neologia, recueillies "à la main "; le deuxième a pris la forme d'une base comportant un programme de repérage automatique, qui deviendra la plateforme Néoveille et le troisième la constitution de différents réseaux internationaux d'observation néologiques s'appuyant sur cet outil.

6 Si le colloque de 2000 sur l'innovation lexicale a été la première initiative visant à rassembler les collègues travaillant sur la néologie, souvent très dispersés, il est loin d'en être la dernière. Jean-François Sablayrolles a organisé plusieurs colloques et journées d'études, sur l'amalgamation lexicale et sur la productivité lexicale, pour ne mentionner que les plus récentes, généralement en collaboration avec l'association ConSciLa (Confrontations en Sciences du Langage). En 2015, le colloque de Cerisy, « La fabrique des mots ", organisé avec Christine Jacquet-Pfau à la suggestion de Michel Arrivé, peut être considéré comme l'apothéose de ces rencontres, réunissant pendant une semaine entière des linguistes de différentes orientations en provenance de plusieurs pays. Les actes ont été publiés l'année suivante chez Lambert-Lucas².

7 Le rayonnement international des travaux de Jean-François Sablayrolles est considérable, d'autant plus qu'il a souvent participé à des manifestations linguistiques dans plusieurs pays lorsqu'il n'était pas invité à y donner des séminaires : au Brésil, en particulier avec Maria Leda Alves, en Espagne avec plusieurs équipes de linguistes, au 
Portugal, le plus souvent à l'initiative de Teresa Lino et dans plusieurs universités d'Espagne et d'Italie. Les coopérations internationales se développaient le plus souvent autour de différents programmes de recherche. Parmi les plus fructueux on peut citer Empnéo qui visait la comparaison de néologie empruntée et «autochtone" dans différentes langues, dont le polonais et le tchèque, sans oublier une étude pilote avec le grec. Ces collaborations internationales ont donné lieu à une suite de publications parues en France, toujours chez Lambert-Lucas (collection "La lexicothèque »), en Pologne et très prochainement en République tchèque. Mais la dimension internationale de ses activités concernait aussi une part importante de formation doctorale car Jean-François Sablayrolles entretenait des relations privilégiées avec ses collègues étrangers, en particulier avec les enseignants algériens, dont plusieurs étaient ses anciens doctorants.

Toujours désireux de consolider l'assise institutionnelle de la néologie, Jean-François Sablayrolles a immédiatement accepté, en 2006, la demande du directeur des Classiques Garnier de fonder une revue de néologie, Neologica, qu'il a dirigée, avec l'auteur de ces lignes, jusqu'en 2018. Il a continué à participer très activement à la vie de la revue, ainsi qu'à celle des Cahiers de lexicographie, dont il faisait partie du comité de lecture ${ }^{3}$, jusqu'à la veille de sa mort. Le numéro de Neologica qui vient de paraître comporte notamment un article qu'il a cosigné. Parmi ses dernières monographies, signalons en particulier Comprendre la néologie. Conceptions, analyses, emplois (Lambert-Lucas, 2019), fait le bilan de toutes ses années de recherche sur la néologie.

Nous avons vu que Jean-François Sablayrolles a participé à de très nombreux colloques et qu'il en a organisé également: ce n'était donc que justice que ses collègues, regroupés autour de Giovanni Tallarico de l'Université de Vérone, aient organisé une rencontre en son honneur, centrée essentiellement sur les derniers programmes européens. Les actes qui ont suivi, publiés également chez Lambert-Lucas, préfacés par Jean Pruvost, comportent bien utilement une bibliographie de l'ensemble des travaux de Jean-François Sablayrolles. Y figurent dans la rubrique "à paraître " plusieurs articles parus depuis, d'autres sous presse (dont celui du présent volume de Linx), sans que la liste soit exhaustive, car Jean-François a travaillé avec acharnement jusqu'à la fin.

10 Jean-François Sablayrolles était donc un chercheur hors pair, qui, suivant la voie qu'il avait très tôt trouvée, a élaboré un programme de recherche cohérent et structuré dans un environnement universitaire où la néologie devait encore faire ses preuves. Mais c'était aussi un collègue attentionné, un professeur attentif et stimulant, bref un homme de qualités humaines et scientifiques exceptionnelles.

\section{NOTES}

1. Pour les références bibliographiques nous renvoyons le lecteur aux «Publications de JeanFrançois Sablayrolles » dans les actes du colloque en son honneur, à savoir : Tallarico, Giovanni, 
Humbley, John, Jacquet-Pfau, C. (dir.), 2020, Nouveaux horizons pour la néologie en français: Hommage à Jean-François Sablayrolles, Limoges, Lambert-Lucas, Collection : « La lexicothèque », p. 245-255.

2. La Fabrique des mots français, Limoges, Lambert-Lucas, coll. « La Lexicothèque », 2016.

3. Voir à ce propos Jacquet-Pfau, C., 2021, « Hommage à Jean-François Sablayrolles », Cahiers de lexicologie, 118, p. 247-251.

\section{AUTEUR}

JOHN HUMBLEY

Université de Paris, CLILLAC-ARP EA 3967 